\title{
DETECTION OF GRATING PATTERNS CONTAINING TWO SPATIAL FREQUENCIES: A COMPARISON OF SINGLE- CHANNEL AND MULTIPLE-CHANNELS MODELS ${ }^{1}$
}

\author{
Norma Graham and Jacob Nachmias \\ Department of Psychology, University of Pennsylvania, Philadelphia, Pennsylvania 19104, U.S.A.
}

(Received 28 March 1970; in revised form 29 June 1970)

\section{INTRODUCTION}

A NEURAL network in which there is excitatory and inhibitory interaction among neighboring units has been proposed as a model of human pattern vision and used, with some success, to explain such phenomena as the appearance of Mach bands and contrast sensitivity with periodic patterns (BéKÉsY, 1960 and RATLIFF, 1965, for example). The network's response is a two-dimensional transformation of the stimulus luminance pattern and is assumed to correspond to the perceived appearance of the pattern. A model of this kind implies that pattern vision is a function of a single neural network, and hence a single transfomation of the stimulus pattern. For this reason we will call it a single-channel model. A single-channel model of some kind underlies all attempts to characterize spatial interactions in human vision by a single modulation transfer function, or equivalently, a single spread function.

Recently CAMPBELL and ROBSON (1968) have suggested a multiple-channels model of pattern vision. This model assumes that many channels simultaneously process the stimulus and that each channel is selectively sensitive to a different narrow range of spatial frequencies. Very roughly, being sensitive to a narrow range of spatial frequencies means responding best to a particular size of element in the pattern; a more precise definition of spatial frequency is given below.

The study reported here compares the predictions of single- and multiple-channels models to results from a psychophysical pattern-detection experiment. The patterns were gratings in which the luminance along any vertical line is constant and the luminance in the horizontal direction varies according to some periodic function. Figure 1 shows two examples of such gratings, along with the functions relating luminance to horizontal distance in each of the two gratings. In the left example, the function is a sinusoid added to a constant luminance (the mean luminance). In the right example, the function is the sum of two sinusoids added to a constant luminance. For gratings such as these, spatial frequency is easily defined: the spatial frequencies contained in a pattern are the frequencies (cycles/ unit distance) of the sinusoids that compose the function relating luminance to horizontal distance. Thus, in the left pattern of Figure 1 , there is one spatial frequency. In the right pattern of Fig. 1, there are two spatial frequencies whose ratio is $3: 1$. (The choice of this ratio of frequencies will be discussed later.) The amount of a component sinusoid at a particular frequency will be expressed by its contrast, where contrast is defined as one half

${ }^{1}$ This work was supported by Grant EY-00302 from the National Eye Institute, National Institutes of Health. The first author was supported by an NSF Graduate Traineeship. Her present address is Rockefeller University, New York, N.Y. 10021, U.S.A. 
the difference between the maximum and minimum luminances due to that sinusoid, divided by the mean luminance. Thus, in the right pattern of Fig. 1, the contrast at the lower frequency is somewhat greater than the contrast at the higher frequency.

Patterns in this study either contained two sinusoidal components whose frequencies were in a 3:1 ratio ( $f$ and $3 f$ ), or they contained only one of the components alone (at $f$ or at $3 f$ ). The former will be referred to as complex gratings, the latter as simple gratings. In the lefthand column of Fig. 2 are shown functions relating luminance to horizontal distance in examples of simple and complex gratings. Two forms of complex gratings differing only in phase were used in our experiments: In the peaks-add form (row 4), the relative phase of the two component sinusoids was such that the peaks of the sinusoid at $f$ are superimposed on peaks of the sinusoid at $3 f$; in the peaks-subtract form (row 3 ), the peaks of the sinusoid at $f$ are superimposed on troughs of the sinusoid at $3 f$.

The last two columns of Fig. 2 show the responses predicted by the two models for the patterns just described. In the single-channel model, the response to a grating containing both component sinusoids will differ from the response to either component alone. In fact, with the assumptions of linearity and symmetry usually made, the response to a grating
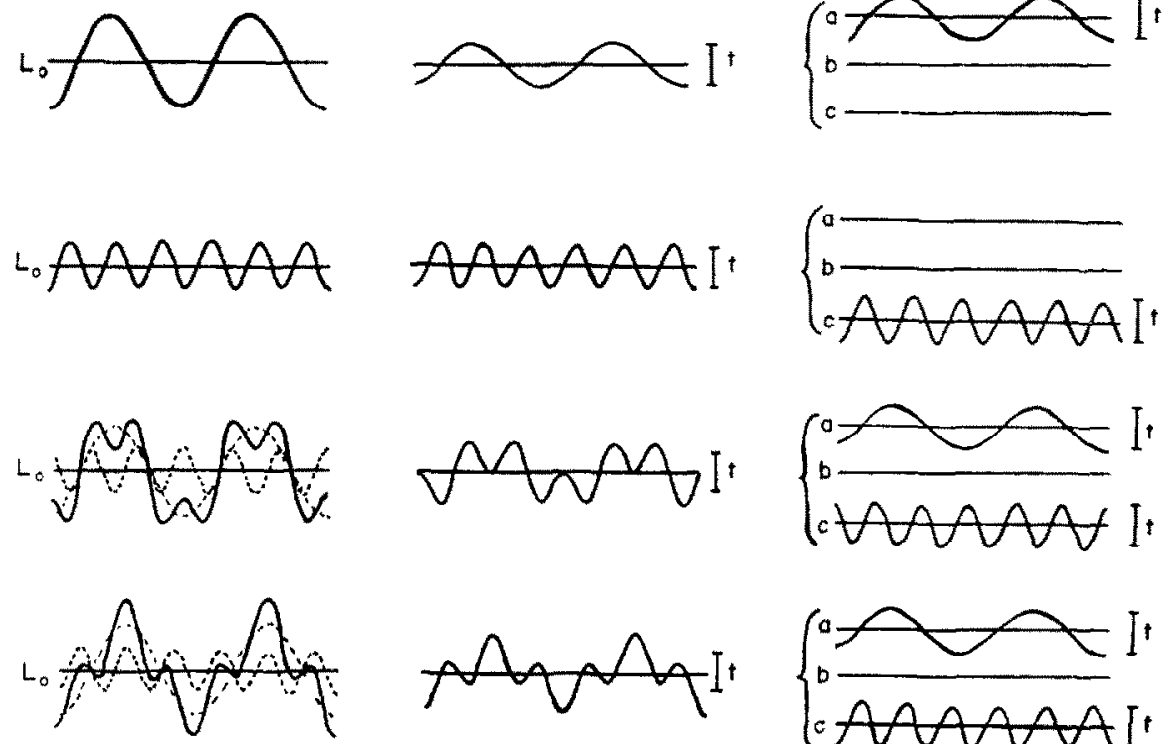

Fig. 2. Four grating patterns and the responses to them predicted by the single-channel and multiple-channels models.

Left column. Functions relating luminance to horizontal distance in two simple gratings (one of frequency three times the other, each at threshold contrast) and two complex gratings (combinations of the above simple gratings in two different phases). The broken lines in rows 3 and 4 indicate the sinusoidal components of the complex gratings (peaks-add phase in row 4, peaks-subtract phase in row 3 ).

Middle column. Function relating response magnitude to distance, according to the singlechannel model.

Right column. Functions relating response magnitude to distance, according to the multiplechannels model. The letters $a, b, c$ refer to channels most sensitive to frequency $f$, to neither frequency, and to frequency $3 f$, respectively. The letter $t$ indicates threshold amplitude. 

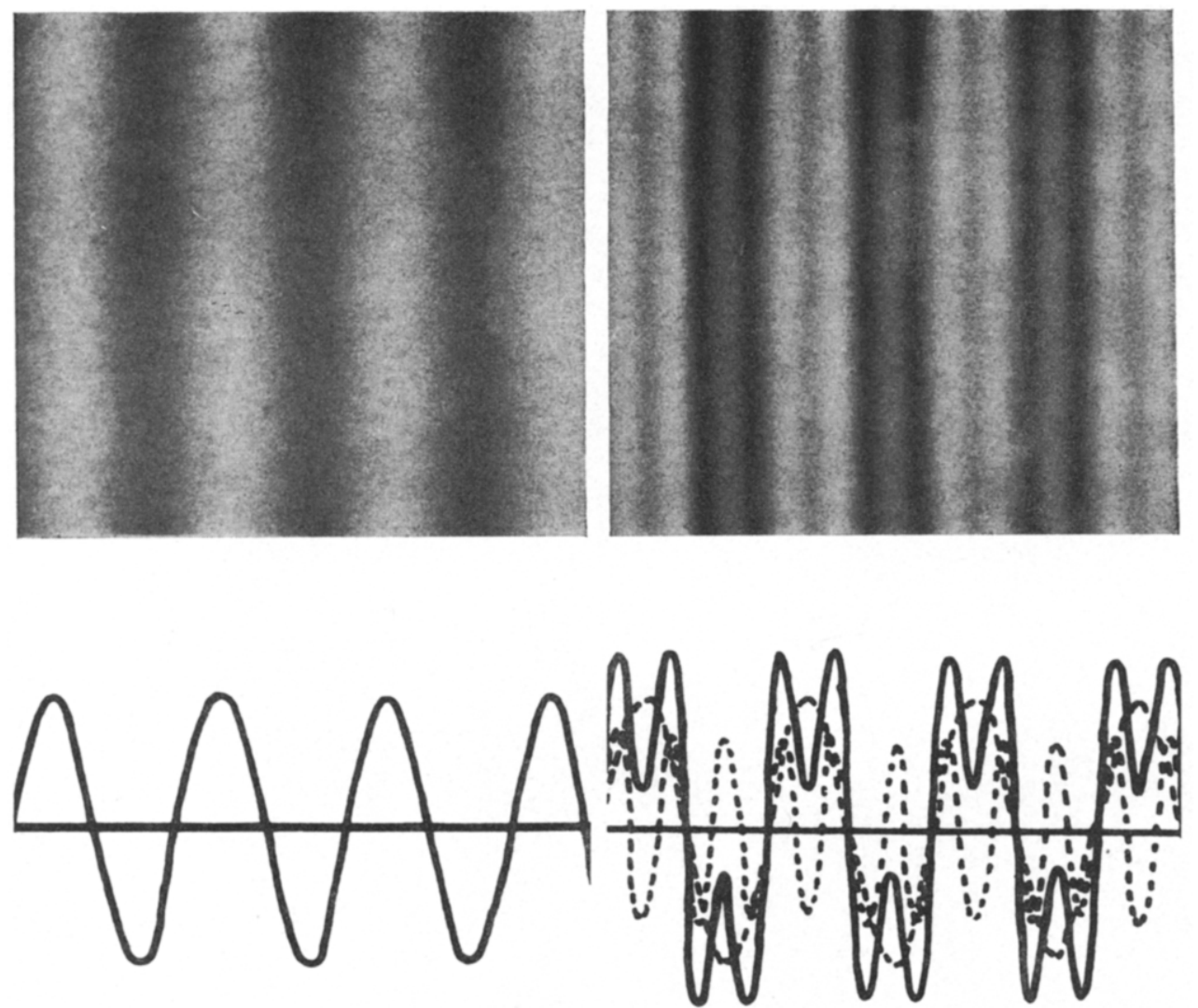

FIG. 1. Examples of the simple and complex grating patterns used in this study. Below each pattern is a graph of the luminance in the horizontal direction across the pattern. The luminance is constant in the vertical direction. The component sinusoids are represented by dotted lines. 
containing both $f$ and $3 f$ is the sum of the responses to the two component sinusoids (which responses are themselves sinusoidal) plus a constant determined by the average luminance. To derive the predictions of the single-channel model for contrast thresholds, some assumption must be made about the criterion for the detection of a pattern. We make the customary assumption that a pattern is detected whenever the largest peak-to-trough difference in the response (or equivalently for these patterns, the largest peak-to-mean difference in the response) is at least as large as a threshold value, $t$.

Assume that the two simple gratings of Fig. 2, column 1 are equally and barely detectable. Then, according to the single-channel model, the response to both of them must contain the same peak-to-trough difference, $t$. On the other hand, in the responses to the two complex gratings, the peak-to-trough difference is unequal, but greater than $t$ in both cases. In fact, for the peaks-add form of grating shown, the peak-to-trough difference in the response is twice the threshold value. To adjust the pattern to threshold (while holding the ratio of contrasts in the two components constant), the contrast in each component must be reduced by a factor of two. In general, according to the single-channel model, the peak-to-trough difference in the response to a peaks-add combination of sinusoids at $f$ and $3 f$ is the sum of the peak-to-trough differences in the responses to the individual components. Thus, for a just-detectable grating of the peaks-add variety,

$$
\frac{\overline{C_{f}}}{\overline{C_{f}^{0}}}+\frac{\overline{C_{3 f}}}{\overline{C_{3 f^{0}}}}=1
$$

where $\overline{C_{f}}$ and $\overline{C_{3 f}}$ are the contrasts of the $f$ and $3 f$ components in the complex grating at threshold and $\overline{C_{f}}{ }^{0}$ and $\overline{C_{3 f}{ }^{0}}$ are the threshold contrasts for the simple gratings.

In the peaks-subtract form of complex grating shown in Fig. 2, row 3, the peak-to-trough difference in the single-channel response is 1.4 times the threshold amount. In general, the magnitude of this difference in the response to peaks-subtract combinations depends on the ratio of contrasts in the components; however, for a large range of ratios, the peak-to-trough difference is substantially less than for the peaks-add combinations. In short, according to the single-channel model, (1) a pattern containing both components will be detectable even though the contrast in each component is substantially below its threshold value when presented alone and (2) the phase in which the two components are added markedly affects the detectability of the combination.

Now consider the multiple-channels model. It postulates the existence of many channels, each sensitive to only a small range of spatial frequencies. If the bandwidth of each channel is sufficiently narrow, no channel will be sensitive to both frequencies $f$ and $3 f$. Responses of three of the channels postulated by this model are shown in Fig. 2, last column: the one that is the most sensitive to $f$ (channel a); the one that is the most sensitive to $3 f$ (channel c); and one of those that is not at all sensitive to either $f$ or $3 f$ (channel b). ${ }^{2}$ As the figure shows, the response in each individual channel to a complex pattern containing sinusoids at $f$ and $3 f$ will be identical to the response of that channel to a simple grating containing one of the sinusoids alone. Note that the phase between the two components does not affect the response of any of the channels shown.

We will assume that a pattern is detected when at least one of the channels contains a

\footnotetext{
${ }^{2}$ Channel $b$ is shown in the figure simply to emphasize that there exist channels other than those that respond to a particular value of $f$ and of $3 f$.
} 
peak-to-trough difference at least as large as a threshold value. Thus, all four patterns of Fig. 2 are detectable according to the multiple-channels model. Now, if the contrast in both components is reduced slightly in either of the complex gratings, the peak-to-trough difference in the response of every channel will be below threshold magnitude. To make precise predictions about the threshold contrasts for complex gratings, we need to know what the correlation is between the noise in the outputs of the different channels. For example, let us assume that the noise is perfectly correlated. Then when the contrast of each component in a complex grating is reduced slightly below threshold for the corresponding simple grating, the pattern as a whole will also be below threshold. (If the noise in the different channels were completely independent, the presence of two components in the pattern would reduce the threshold because of probability summation.) Thus the bottom two patterns shown in Fig. 2 are exactly at threshold according to a correlated-noise version of a multiple-channels model. In general, according to this model, a pattern containing two components of different frequencies will be at threshold if, and only if, one of the components is at its own threshold contrast, regardless either of the phase between components or of the contrast in the other component, provided it is below threshold.

CAMPBELL and RoBSON (1964) briefiy report an experiment similar to our own. They state, "When a second harmonic was added to a sine-wave grating (in any phase relation), it appeared to be detected independently". We chose to use components whose frequencies were in a ratio of $3: 1$ because (a) they are less likely to fall within the effective bandwidth of any one channel, if the multi-channel model is correct, and (b) they give rise to larger phasedependent response differences, if the single-channel model is correct.

\section{METHODS}

\section{Apparatus}

The general method of generating patterns on the face of a CRT has been described by CAMPBELL and GRERN (1965). To produce a sinusoidal luminance variation at spatial frequency $f$, the output of a freerunning oscillator (General Radio 1309-A) was used to modulate the intensity of the CRT's electron beam, as well as to synchronize its sweep. The same signal also served to synchronize another function generator (Wavetek Model 116) in both frequency and phase. This second generator produced a square-wave signal whose frequency was exactly the same as that of the input signal, and whose relative phase could be set by the experimenter. A third oscillator (General Radio 1310-A) was synchronized in frequency and phase to the third harmonic in the square wave, giving rise to a signal which could be used to produce sinusoidal luminance variation on the CRT at frequency $3 f$.

The contrast of the pattern was varied and also recorded by means of a recording attenuator (GrasonStadler). At all times during a session, whether or not there was a pattern present, the mean luminance of the CRT face was constant at $3 \mathrm{ft}-\mathrm{L}$, except in experimental sessions with $f=1.8$ cycles/deg, when it was $5 \mathrm{ft}-\mathrm{L}$. The CRT had a $P 31$ phosphor, and thus looked yellowish-green in color.

The edges of the CRT screen were masked, exposing a rectangular area which at the viewing distance of $103 \mathrm{~cm}$, subtended 4.8 by 4.4 deg of visual angle (except that, in the conditions where the lower frequency was 0.9 or $1.8 \mathrm{cycles} / \mathrm{deg}$, the whole screen of approximately $6^{\circ}$ dia. was exposed). To help the subject maintain fixation and accomodation, diagonal cross-hairs were placed just in front of the screen. The subject viewed the screen through an artificial pupill $1.5 \mathrm{~mm}$ dia. while biting on a dental impression board to hold his head in position.

\section{Procedure}

In each experimental session, the lower frequency, $f$, was set at one of seven values ranging from 0.9 to 6.3 cycles/deg. For each value of $f$, contrast thresholds were measured for the two simple gratings of frequency $f$ and $3 f$ respectively, and for the two forms (peaks-add and peaks-subtract) of complex gratings. During each threshold determination with either complex grating, the ration of contrast at $f, C_{f}$, to contrast at $3 f, C_{3} f$, was kept constant at one of three values: $1 / 2,1$ or 2 times the ratio of the threshold contrasts for the corresponding simple gratings. That is,

$$
\left(C_{f} / C_{3 f}\right) \div\left(\overline{\left.C_{f}^{0} / \overline{C_{3 j}}\right)}=1 / 2,1 \text {, or } 2\right.
$$


At the start of each experimental session, preliminary estimates of $\overline{C_{f}{ }^{\circ}}$ and $\overline{C_{3 f}{ }^{\circ}}$ (threshold contrasts for the simple gratings) were obtained for the purpose of setting the desired ratios of $C_{f}$ to $C_{3 f}$. Contrast thresholds for the simple gratings were redetermined during the experimental session proper, along with those for the complex gratings. Contrast thresholds were determined by two psychophysical procedures, (a) adjustment and (b) temporal forced-choice staircase. Thresholds for gratings at two values of $f$ were measured by both methods; the rest were measured only by one or the other.

In the adjustment procedure, the observer varied the contrast until he could "just see a pattern" by pushing one button to increase and one to decrease the contrast. The initial contrast for each adjustment was chosen randomly from a set spanning two log units. As long as the subject held a button down, the contrast of the pattern changed at the rate of $0.4 \mathrm{log}$ unit/sec. Each adjustment to threshold took between 5 and $20 \mathrm{sec}$. When the adjustment procedure was used, thresholds for each of the grating patterns at a particular value of $f$ were all measured within one session; each pattern's threshold was determined between ten and 12 times, depending on how many adjustments could be comfortably made in that session (with the exception noted below for $f=2.7 \mathrm{cycles} / \mathrm{deg}$ ).

In the temporal forced-choice staircase procedure, a staircase consisted of 405 -sec trials. Each trial contained two intervals, signalled by clicks. The pattern was presented for $750 \mathrm{msec}$ during one of these two intervals chosen at random. The subject responded by pushing one of two buttons to indicate which interval he thought contained the pattern. If he was correct, a tone was sounded. The contrast in the pattern varied from trial to trial in the staircase by the following rule: decrease the contrast one step if the subject has just been correct twice in a row at the present contrast level; increase the contrast one step whenever he has been incorrect. One step represented a change of contrast by a factor of $1.26(0.1 \mathrm{log}$ unit), except that, because of a peculiarity in the construction of the recording attenuator, a step which was opposite in direction to the one preceding it represented a change by a factor of $1.12(0.05 \mathrm{log}$ unit).

A threshold estimate was obtained from each staircase by taking the mean of the log contrast values for those trials after which the direction of contrast change was reversed. When the forced-choice procedure was used, the threshold for each pattern was determined six times, twice on each of 3 days (except for $f=$ 2.7 cycles $/ \mathrm{deg}$ ). For $f=2.7 \mathrm{cycles} / \mathrm{deg}$, seven staircase and nine adjustment determinations of the thresholds for each pattern were intermixed over five sessions.

At the beginning of each session, the observer was dark adapted for at least $5 \mathrm{~min}$, and then adapted to the mean luminance of that session for at least $1 \mathrm{~min}$ before any observations were made.

\section{RESULTS}

The results are summarized in Fig. 3. The coordinates are $\overline{C_{f} / \bar{C}_{f}^{0}}$ and $\overline{C_{3 f} / \bar{C}_{3 f}{ }^{0}}$, namely the contrast of each component in a complex grating at detection threshold, relative to the threshold contrast of the corresponding simple grating. ${ }^{3}$ In plotting the results, the values of $\overline{C_{f}{ }^{0}}$ and $\overline{C_{3 f^{0}}}$ were taken to be those obtained in the body of the experiment rather than the preliminary estimates used for setting the ratios of $C_{f}$ to $C_{3 f}$. Consequently, the ratio of the coordinates of each plotted point is not necessarily $1 / 2,1$ or 2 . Thresholds for peaks-add patterns are indicated by capital letters; thresholds for peaks-subtract patterns are indicated by small letters. For each point, the frequency, the subject, the procedure and the number of determinations that entered into the plotted average threshold are given in the figure legend. The standard error of the mean of each average threshold was between $41 / 2$ per cent $(0.02$ $\log$ units) and 20 per cent $\left(0.08 \mathrm{log}\right.$ units) with an average of 10 per cent $(0.04 \log$ units $){ }^{4}$

Also plotted in Fig. 3 are the outcomes of this experiment predicted by the singlechannel and multiple-channels models. On the single-channel model, thresholds for the

\footnotetext{
${ }^{3}$ The actual values of contrast thresholds for simple gratings are not important for the purpose of this study and so are not reported in detail. The values for subject JK, when plotted against frequency, form a contrast sensitivity curve similar to those presented by CAMPBELL and ROBSON (1968) in their Fig. 3. The minimum value of threshold contrast was 0.44 per cent and occurred at 3.6 cycles/deg.

- It should be emphasized that although the results are displayed in Fig. 3 on linear coordinates for ease of comparison with the models' predictions, all sample statistics, such as means and standard errors, were computed on the logarithms of the estimated values of threshold contrasts. We performed the logarithmic transformation primarily in order to satisfy the assumptions of normality and equal variance required by our statistical tests. Nevertheless, for a portion of our results we did calculate sample statistics in terms of both contrast and log contrast. Both methods of calculation led to the same conclusions.
} 


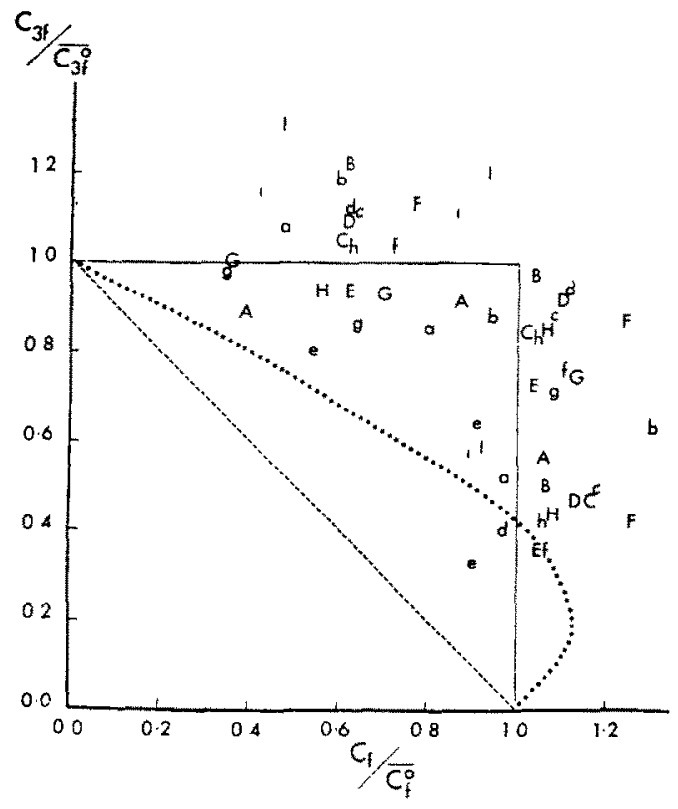

Fig. 3. Contrast thresholds for complex grating patterns containing two frequencies, $f$ and $3 f$, in two different phases. The coordintes are the contrast of each component in the complex grating at threshold relative to the threshold contrast of the corresponding simple grating. Results obtained with the peaks-add form of complex gratings and with the peaks-subtract form are plotted as capital and small letters, respectively. The corresponding predictions of the single-channel model are represented by the diagonal dashed line and the dotted curve. The upper and right edges of the square represent the predictions of the multiple-channels model for both peaks-add and peaks-subtract gratings.

\begin{tabular}{ccccc}
\hline Symbols & $\begin{array}{c}\text { Frequency }(f) \\
\text { cycles/deg }\end{array}$ & Method & Subject & $\begin{array}{c}\text { Number of } \\
\text { determinations }\end{array}$ \\
\hline A,a & 0.9 & Staircase & JK & 6 \\
B,b & $1 \cdot 8$ & Staircase & JK & 6 \\
C,c & $2 \cdot 7$ & Staircase & JK & 7 \\
D,d & $2 \cdot 7$ & Adjustment & JK & 9 \\
E,e & 3.6 & Staircase & PA & 6 \\
F,f & 3.6 & Adjustment & JK & 10 \\
G,g & 4.5 & Adjustment & JK & 12 \\
H,h & 5.4 & Adjustment & JK & 10 \\
I,i & 6.3 & Adjustment & JK & 12 \\
\hline
\end{tabular}

peaks-add patterns should lie along the diagonal line, $\overline{\left(C_{f} / \overline{C_{f}}\right)}+\left(\overline{\left.C_{3 f} / C_{3 f}{ }^{0}\right)}=1\right.$, and thresholds for peaks-subtract patterns should lie along the dotted curve. According to the correlated-noise version of the multiple-channels model, thresholds for all complex gratings

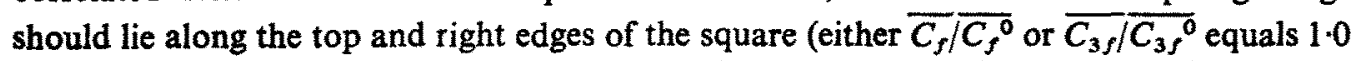
when the other ratio is less than or equal to 1.0 ). It is apparent that the multiple-channels model fits the experimental results much better than does the single-channel model. There do not appear to be any consistent differences in the results that can be attributed to 
differences of absolute spatial frequency, mean luminance, psychophysical procedure, or subjects. Therefore, the results from all the experiments represented in Fig. 3 will be considered together.

The predictions of the two models were also evaluated statistically by means of $t$-tests. The differences were calculated between the average log threshold contrasts for each pair of complex gratings that differed only in phase. According to the single-channel model, the threshold of the peaks-subtract form should be at least 30 per cent (or 1/8 of a log unit) larger than the threshold for the peak-add form, for pairs of gratings in which the ratio of relative contrast of $3 f$ to relative contrast at $f, \overline{\left(C_{3 f} / C_{3 f}{ }^{0}\right)} \div \overline{\left(C_{f} / C_{f}{ }^{\circ}\right)}$, is between $1 / 4$ and 1 . (When this ratio lies outside the range, the predicted difference is less, and so more like the multiple-channels predictions.) Of the 15 pairs of patterns whose contrast fell within this range, 13 yielded differences in threshold that were significantly smaller than the predicted minimum value of 30 per cent (at the 0.05 level of confidence, one-tailed test). Thus the expectation of greater detectability of peaks-add than of peaks-subtract patterns, based on the single-channel model, is not supported. On the other hand, the multiple-channels model predicts that complex gratings differing only in phase will be equally detectable. Twenty-four of the 27 differences were not significantly different from zero (at the 0.05 level, two-tailed test). Three exceptions out of 27 are not unexpected at the 0.05 level. These results are therefore consistent with the multiple-channels model.

The single-channel model predicts a linear relation between $\overline{C_{f} / C_{f}{ }^{0}}$ and $\overline{C_{3 f} / C_{3 f}{ }^{0}}$ for peaks-add patterns:

$$
S=\frac{\overline{C_{f}}}{\overline{C_{f}^{0}}}+\frac{\overline{C_{3 f}}}{\overline{C_{3 f}}}=1 .
$$

In Fig. 3 it is quite clear that the peaks-add points do not lie on the diagonal. As might be expected, all peaks-add patterns produced values of $S$ significantly greater than one, according to a $t$-test incorporating an approximate estimate of the variance of $S$.

All points in Fig. 3 lie near the lines $\overline{C_{f}} / \overline{C_{f}^{0}}=1$ and $\overline{C_{3 f} /} \overline{C_{3 f^{0}}}=1$, predicted by the multiple-channels model. More precisely, the lines lie within the 95 per cent confidence intervals around the larger coordinates of each of the 54 points, with the exception of only three points. (For these three patterns, the larger coordinates were actually significantly greater than 1.0 .) Overall, the fit of the multiple-channels model seems satisfactory. ${ }^{5}$

\section{DISCUSSION}

As was discussed in detail in the introduction, a single-channel model of pattern vision predicts that the phase between two components in a complex pattern will affect the detectability of that pattern; also, in many cases, complex patterns should be detectable even though the contrast in each component is substantially below the threshold contrast for that component presented alone. A multiple-channels model predicts insensitivity to phase between components. In addition, according to the correlated-noise version of the multiplechannels model, a complex pattern (containing two quite different frequencies) will be

\footnotetext{
5 The scatter of points in Fig. 3 actually is not as bad as might appear at first glance. Note that the points are plotted on linear, rather than on logarithmic coordinates. Also, recall that the lines predicted by the multiple-channels models lie within the 95 per cent confidence intervals of 51 out of 54 points. Furthermore, the average of the standard errors on which these confidence intervals are based is only 0.04 log unit (10 per cent), not an uncommonly large value for this type of psychophysical measurement.
} 
detectable if and only if the contrast in at least one of the components is at or above the threshold for that component presented alone.

In this study, we tested the models by measuring contrast thresholds for patterns containing two sinusoidal components, one of frequency three times the other, added in two different phase relations. Our results clearly contradict the single-channel model and support the multiple-channels model. Results from the patterns containing a particular lower frequency $f$ can only provide evidence for the existence of two channels, but the results from all frequencies tested argue for the existence of at least three and probably more channels in the $6^{\circ}$ central area of retina stimulated.

In order to derive exact predictions from the two models, we adopted some additional assumptions about their other properties. We must now consider whether our conclusions about the models are critically dependent upon these auxiliary assumptions. For example, in the case of the single-channel model, we assumed linearity, at least for near-threshold contrasts. Conceivably by abandoning this particular assumption in favor of some other one the single-channel model could be made to account for our results. (However, we have not discovered such an assumption.) On the other hand, our results cannot be taken to support the correlated noise version of the multiple-channels model in particular. If the noise in the several channels were not perfectly correlated, then the predicted function, when plotted on the coordinates of Fig. 3, would have a rounded, rather than a sharp corner on the upper right. The extent of rounding would depend upon the amount of noise and the extent of its correlation. Clearly our results are compatible with a predicted function which has a moderately rounded corner.

Previous studies (Blakemore and Campbell, 1969; Pantle and Sekuler, 1968) have shown that looking at a high-contrast grating of one spatial frequency will raise the contrast threshold for gratings of that and similar frequencies, but will not affect the contrast threshold for gratings of very different frequencies. This evidence strongly implies that channels selectively sensitive to different narrow ranges of spatial frequencies exist at some level in the visual system. However, this evidence does not show that these channels act separately in the detection of patterns containing more than one frequency. It is conceivable that separately adaptable channels exist in the visual system, but that their outputs combine before the level where detection of complex patterns is determined. CAMPBELL and RoBSON (1968) suggested that the outputs of these channels are actually detected separately. As evidence for this suggestion, they reported thresholds for the detection of sinusoidal, square-wave and other periodic gratings, as well as thresholds for discrimination between sine- and square-wave gratings. Unfortunately, the difference between the two models' predictions for such patterns is appreciable only for patterns of low spatial frequency. And in that low-frequency range, Campbell and Robson's results are not well fit by either the single-channel or multiple-channels models. The results of the present study are consistent with the multiplechannel model for detection and contradict the single-channel model. Thus this study supports the hypothesis that channels exist in the visual system that are selectively sensitive to different narrow ranges of spatial frequency and that these channels act separately in the detection of complex patterns.

Another piece of evidence supporting the hypothesis of separate channels comes from a study by NACHMIAS, SACHS and RoBson (1969). They obtained concurrently the psychometric functions for simple and for complex gratings, the relative frequency of whole components was varied over a wide range. Their aim was to test the hypothesis that the two components of a complex grating can be detected independently. When the two components 
differ enough in frequency to be detected only by separate channels, this hypothesis follows from a multiple-channels model in which the variability in the different channels is completely uncorrelated. The hypothesis was supported for complex gratings in which the component frequencies differed by a factor of at least 2 , and rejected for complex gratings in which the frequencies differed by a factor of $1 \cdot 25$.

\section{REFERENCES}

Blakemore, C. and Campbell, F. W. (1969). On the existence of neurones in the human visual system selectively sensitive to the orientation and size of retinal images. J. Physiol. 203, 237-260.

CAmprell, F. W. and Green, P. G. (1965). Optical and retinal factors affecting visual resolution. J. Physiol. 181, 576-593.

Campbell, F. W. and Robson, J. G. (1964). Application of Fourier analysis to the modulation response of the eye. J. opt. Soc. Am. 54, 581A.

CAmpbell, F. W. and Robson, J. G. (1968). Application of Fourier analysis to the visibility of gratings. J. Physiol. 197, 551-566.

NACkMiAS, J., SACHS, M. B. and RoBSON, J. G. (1969). Independent spatial frequency channels in human vision. J. opt. Soc. Am. 59, 1538A.

Pantle, A. and Sexuler, R. (1968). Size-detection mechanisms in human vision. Science, N. Y. 162, 11461148.

RATLIfF, F. (1965). Mach Bands: Quantitative Studies on Neural Networks in the Retina, Holden-Day, San Francisco.

voN BÉkÉsY, G. (1960). Neural inhibitory units of the eye and skin. Quantitative description of contrast phenomena. J. opt. Soc. Am. 31, 1236-1249.

Abstract-Contrast thresholds were measured for gratings containing two superimposed sinusoidal components. The frequency of one component was always three times that of the other, but the phase between components and the ratio of their contrasts took on several values. Two models of pattern vision were tested (1) a single-channel model in which pattern vision is a function of a single neural network and (2) a multiple-channels model in which the stimulus information is processed by many channels, each sensitive to a narrow range of spatial frequencies. Results support the multiple-channels and reject the single-channel model.

Résumé-On mesure les seuils de contraste avec des réseaux contenant deux composantes sinusoidales superposées. La fréquence d'une composante est toujours triple de l'autre, mais la phase entre les composantes et leurs rapports d'amplitudes peutent prendre diverses valeurs. On teste deux modèles de vision des formes: (1) un modèle à canal unique où cette vision n'est fonction que d'un seul réseau nerveux, et (2) un modèle à canaux multiples où l'information sur le stimulus est transmise par plusieurs canaux, chacun étant sensible dans un domaine étroit de fréquences spatiales. Les résultats sont en faveur des canaux multiples et condamnent le modèle à canal unique.

Zusammenfassung-Es wurden Kontrastschwellen für Gitter gemessen, welche zwei einander übergesetzte Sinuskomponenten enthielten. Die Frequenz einer der Komponenten war immer dreimal so gross als die andere, aber ihr Phasenunterschied und das Kontrastverhältnis nahmen verschieden Werte an. Es wurden zwei Modelle des Mustersehens geprüft: (1) ein Einbahnmodell, in welchem das Mustersehen eine Funktion eines einzigen Nervennetzwerkes ist und (2) ein Vielbahnmodell, in welchem die Reizinformation auf mehreren Wegen verarbeitet wird, deren jeder auf einen engen Raumfrequenzbereich empfindlich ist. Die Ergebnisse stützen das Vielbahnmodell und widersetzen sich der Einbahnidee.

Резюме-Выли измерены контрастные пороги для решётки, содержашей два налагаюшихся синусондальных компонента. Частота оаного компонента всегда отличалась от частоты другого в три раза, но фазы мехду компонентами и соотношение их контрастов имели несколько значения. Здесь были нспытаны две модели паттернов зрения: (1) одноканальная модель, в которой паттерн зрения является фунхиеи единой нервной сети, в (2) многоканальная модель, в которой информация о стимуле передается многими каналами, из которых кахдын чувствителен х узкому диалазону просторанствен ных частот. Полученные результаты поддержнвают мультиханальную модель и позвсляют отбросить одноканальную. 